\title{
TENS to the Lateral Aspect of the Knees During Stance Attenuates Postural Sway in Young Adults
}

\author{
Yocheved Laufer* and Ruth Dickstein \\ Department of Physical Therapy, Faculty of Social Welfare and Health Studies, University of \\ Haifa, Haifa, Israel \\ E-mail: yocheved@research.haifa.ac.il
}

Received August 15, 2007; Revised September 29, 2007; Accepted September 30, 2007; Published November 26,2007

\begin{abstract}
Somatosensory input is known to be essential for postural control. The present study examined the effects on postural sway of sensory input delivered via transcutaneous electrical nerve stimulation (TENS) applied to the knees during stance. Electrodes from a dual-channel portable TENS unit were adhered to the skin overlying the lateral and medial aspect of both knees of 20 young healthy volunteers (mean age 24.0 years, standard deviation 4.0). Postural sway parameters were obtained during static bipedal stance with an AMTI force platform. Four stimulation conditions were tested with eyes open and with eyes closed: no TENS; TENS applied bilaterally; and TENS applied to either the right or the left knee. Participants underwent two eight-trial blocks, with each trial lasting 30 seconds. The order of conditions was randomized for each participant. Stimulation consisted of a biphasic symmetrical stimulus delivered at the sensory detection level, with a pulse duration of $200 \mu \mathrm{sec}$ and a pulse frequency of $100 \mathrm{~Hz}$. The application of TENS induced significant reductions in mean sway velocity and in the medio-lateral dispersion of the center of pressure, with no corresponding effect on the anterior-posterior dispersion. These findings suggest that electrical stimulation delivered at the sensory detection level to the lateral aspects of the knees may be effective in improving balance control, and that this effect may be directionally specific.
\end{abstract}

KEY WORDS: TENS, posture, stance, stability, child health \& human development, neuroscience, sensation and perception

\section{INTRODUCTION}

Sensory information provided by the visual, somatosensory and vestibular systems are essential for postural control during stance. Ongoing somatosensory input required for postural control is mainly provided by ankle proprioceptors[1,2], cutaneous receptors in the sole of the foot $[3,4,5,6,7]$, as well as receptors in the knee, hip, trunk, and cervical spine joints and muscles[8,10]. Augmentation of somatosensory input during stance is often needed, such as when stance is challenged by external interferences or when the sensory systems that sub serve postural control are affected by pathology[8,9,10,11,12]. For example, individuals with peripheral sensory neuropathy may utilize somatosensory input from their fingertips by light touch in order to compensate for their lower extremity proprioceptive deficits[13]. 
The application of somatosensory input to enhance postural stability is routinely performed during clinical rehabilitation, primarily through manual contact. The advantage of utilizing these maneuvers is determined by the ability to adjust them in accordance with patient needs. However, manual input is not consistent and the gauging of factors related to its delivery is virtually impossible, given that the amplitude, frequency, and duration of manual contact are not amenable to routine quantification. Furthermore, as manual contact is dependent on direct interaction between the person providing the input and the person in need of somatosensory enhancement, the ability to offer ongoing assistance is limited by practical constraints.

Transcutaneous electrical nerve stimulation (TENS), which involves the pulsatile stimulation of sensory fibers, is a widely applied clinical modality used primarily for the purpose of pain modulation[14,15,16]. Studies have shown that TENS-induced analgesic effects are related to decreases in the activity of noxiously evoked dorsal horn cells stemming from the activation of cutaneous and deep tissue afferent fibers[17,18]. TENS has also been shown to affect upper motor neuron and motor cortex excitability[19,20], which manifests clinically in decreases in spasticity among patients with post-stroke hemiparesis[21,22], and multiple sclerosis[23]. Sensory stimulation also appears to enhance the recovery of functional stability in patients following a stroke[24,25]. Furthermore, the application of TENS to the neck muscles in patients with hemispatial neglect has been shown to improve spatial orientation and postural control[26,27,28,29].

Yet, while TENS is a highly accessible and easily applied modality shown to provide effective somatosensory input modulating central neural activity, its effect on postural stability has rarely been the focus of research attention. In our previous study conducted on this topic, we applied stimulation to the skin overlying the posterior calf musculature, resulting in the attenuation of postural sway as expressed by mean sway velocity[30]. The aim of the current study was to gain further insight into the effects of sensory TENS on stance stability in healthy individuals by applying threshold sensory stimulation along the medio-lateral axis at the knee level. Stimulation at this site was selected on the basis of a previous study in which the application of random electrical noise applied at this location brought about a decrease in postural sway, specifically in the medio-lateral plane[31]. Thus, based on our own as well as on the later cited work, we hypothesized that the administration of TENS would be associated with a decrease in postural sway, with the effect being especially pronounced in the medio-lateral plane. Increasing stability in this plane is of particular interest, as it has been shown to be associated with instability in various pathological conditions[32,33].

\section{METHODS}

Twenty university students (6 men and 14 women), with a mean age of 24.0 years (standard deviation 4.0), volunteered to participate in the study. Participants were blind as to the study objectives. With the exception of one individual, all participants had right leg dominance. Individuals with a history of pathology affecting one of the lower limbs and/or the postural control system (e.g., vestibular disorders) were excluded. The research was approved by the Institutional Ethical Review Committee, and all participants signed an informed consent form in which their rights as subjects were clearly outlined.

\section{Instrumentation and Protocol}

The study was conducted during the morning hours in a quiet room. Prior to testing, adhesive TENS electrodes $(2.5 \times 2 \mathrm{~cm})$ were fastened to the skin overlying the lateral and medial aspect of both knee joints, with the midline of the electrodes positioned parallel to the joint gap line. Except for the specific stimulation sites, the general protocol was similar to the one applied in our previously reported study[30]. Testing was conducted with participants standing in their socks on a portable 50x50x3cm AMTI force plate (Advanced Mechanical Technology Inc., 176 Waltham St, Watertown, MA 02472, USA), while maintaining an 8cm distance between the medial malleoli and a $10^{\circ}$ angle between their feet. When assuming the testing position 
for the first time, the circumference of the feet was marked on the plate in order to assure participants' return to the same foot placement in each trial. The participants' upper extremities were kept folded against their chest throughout all testing trials.

The testing procedure consisted of two blocks of eight trials, with each trial lasting 30 seconds, and a resting period of three minutes between trial blocks. A unique combination of a visual condition and a stimulus condition was used in each trial, and the order of conditions was randomized for each participant. The two visual conditions were: eyes open (EO) and eyes closed (EC). The four stimulation modes were: No TENS (NT); TENS applied bilaterally (BT); TENS applied only to the left knee (TL); and TENS applied only to the right knee (TR).

The stimulation was delivered by a dual-channel battery-operated commercial TENS unit (Elpha 2000 unit, 301 Moodie Drive, Suite 205 Ottawa, Ontario K2H 9C4 Canada). Stimulation consisted of a biphasic symmetrical pulse, with a $200 \mu \mathrm{sec}$ pulse duration and a $100 \mathrm{~Hz}$ frequency. Stimulation amplitude was adjusted before the start of each trial in one-milliampere increments and was set at the sensory detection threshold of each participant.

\section{Data Analysis}

Center of pressure (COP) data were collected at a $50 \mathrm{~Hz}$ sampling frequency and analyzed offline by a dedicated software program (AMTI Accuswayplus). Postural control was assessed with the following variables: mean COP velocity, which represents the total distance traveled by the COP divided by testing time; amplitude variability of the COP excursion in the anterior-posterior direction; and amplitude variability of the COP excursion in the medio-lateral direction.

For each variable, the mean values of identical trials in the two blocks were used for analysis. Descriptive statistics and repeated measures ANOVA were applied to compare the effects of the four TENS conditions (i.e., NT, BT, TL, TR) and to examine their potential interactions with the effects of vision (i.e., EO, EC). Significance was set at $p<0.05$. Preplanned contrasts were further applied to test the effects of NT (No Tens) against the pooled effect of the other three TENS conditions.

\section{RESULTS}

No significant interactions were observed between the visual conditions and the TENS conditions. Visual condition had a significant effect on all the examined variables, with larger mean sway velocity and sway variability found in both the ML and AP directions for the eyes closed condition than for the eyes open condition. Taken individually, none of the TENS conditions had a significant effect on mean sway velocity. However, the pooled effect of the TENS conditions, as compared to the No TENS condition, resulted in a significant TENS-induced decrease in mean sway velocity $(\mathrm{F}(1,18)=7.29, \mathrm{p}=0.01$, partial $? 2=0.046)$. The mean sway velocity in each of the four TENS conditions is depicted in Figure 1. 


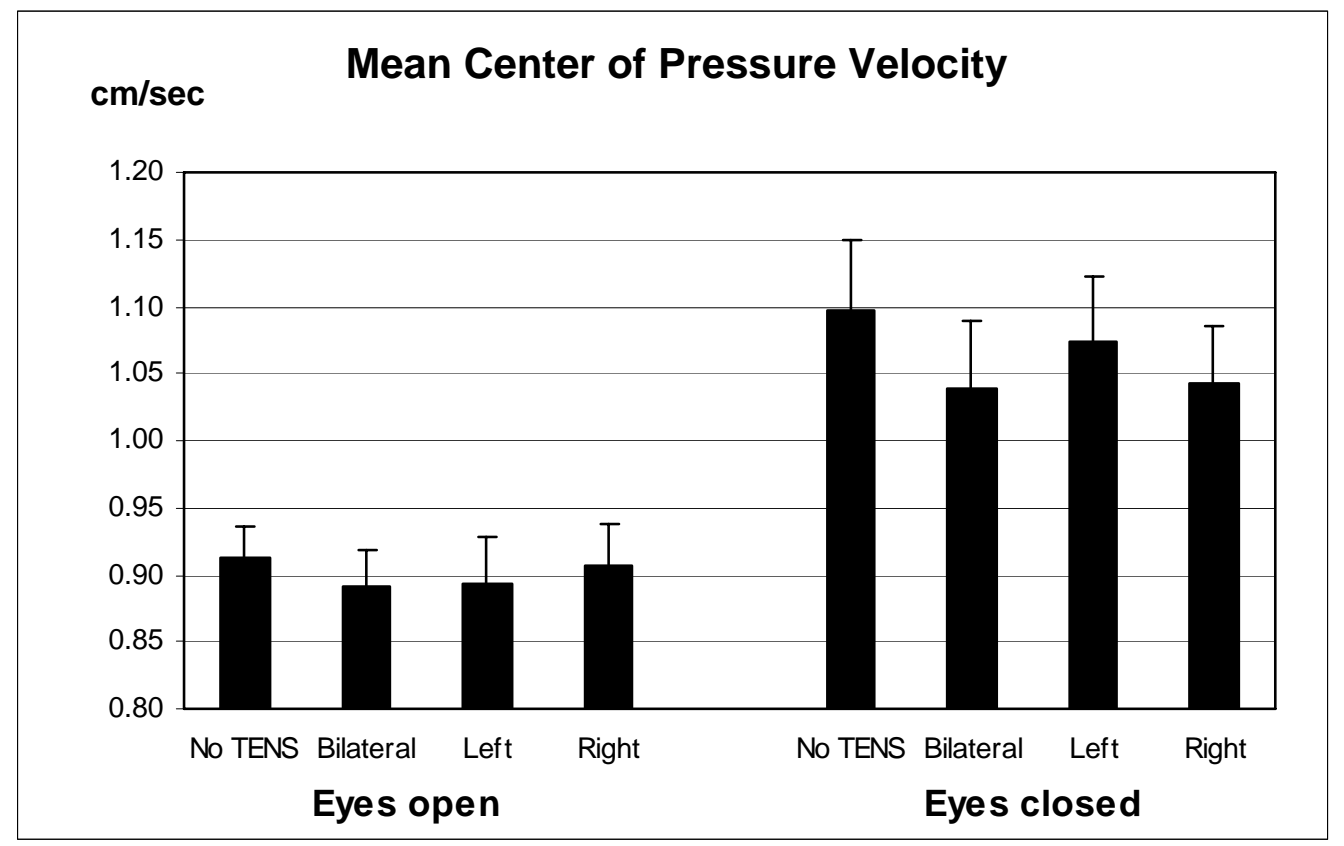

Figure 1. Mean and standard deviation of center of pressure sway velocity in each of the four TENS conditions with eyes open and eyes closed.

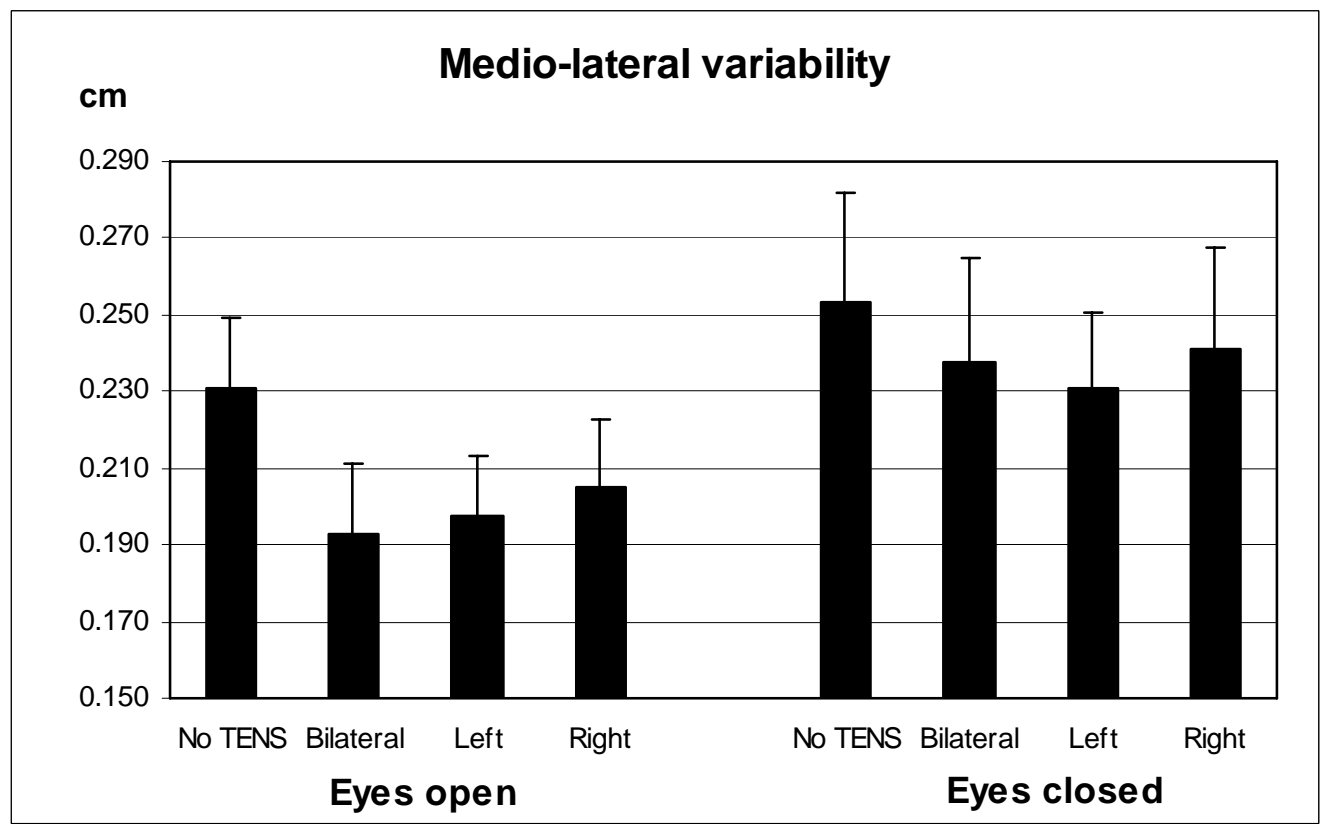

Figure 2. Mean and standard deviation of medio-lateral variability in each of the four TENS conditions with eyes open and eyes closed.

A similar trend is noted for the effect of the TENS conditions on medio-lateral variability $(F(3,16)=2.58$, $\mathrm{p}=0.089$ ), with their pooled effect resulting in a significant TENS-induced decrease, as compared to the No TENS condition $(\mathrm{F}(1,18)=6.81, \mathrm{p}=0.018$, partial $? 2=0.04)$. No similar TENS-induced effect was observed 
in the anterior-posterior direction. The medio-lateral variability in each of the four TENS conditions is depicted in Figure 2.

\section{DISCUSSION}

The present study demonstrated that COP mean velocity during bipedal static stance is attenuated by TENS applied to the medial and lateral aspects of the knees at sensory threshold intensity. Moreover, the present treatment protocol reduced COP sway variability solely along the frontal plane. In accordance with previous studies, visual input contributed significantly to stability[34,35], with the observed directionally specific effect of TENS found to be independent of the visual condition.

COP movement, expressed as path length (or its equivalent mean velocity), has been determined as the most sensitive postural measure for differentiating between stance conditions or age groups[36]. Yet, changes in this variable alone do not necessarily imply changes in level of stability[37]. More relevant to stability are sway variability measures in the anterior-posterior and/or the medio-lateral directions, which signify the amplitude of the movement around the mean COP location. Decreases in amplitude variability point to a stiffening strategy, which is observed during stance under conditions perceived as threatening to stability, such as when standing on a raised surface[38,39] or while performing a secondary cognitive task demanding attention[40]. It is hypothesized that the prioritization of stability achieved by reducing the amplitude of the sway allows attention to be diverted to whatever secondary condition may be affecting postural control.

The unique finding of the current study lies in the demonstration of a direction-specific effect of the TENS application, as determined by decreases in sway variability in the medio-lateral plane. Human joint movements and related muscular activity are largely expressed along the two orthogonal planes, namely, the anterior-posterior and the medio-lateral planes. It is intuitively assumed during rehabilitation that manually applied somatosensory cues facilitate stability along the plane of sensory input. Thus, for example, when an individual exhibits a tendency to sway/fall sideways, sensory cues are generally provided along the lateral aspects of the body.

In our previous work[30], the application of TENS posteriorly to the skin overlying the medial and lateral gastrocnemius muscles demonstrated a similar reduction in overall sway, but did not induce a similar directionally specific effect. Control of stability in the medio-lateral axis is of particular clinical significance, given that it is affected by a wide range of disorders. For example, elderly fallers, as well as individuals with hemiparesis or Parkinson's disease, manifest increased medio-lateral instability[32,33]. Yet, the clinical significance of the observed reduction in sway amplitude has yet to be determined.

While these results support the intuitive approach of applying sensory cues in the direction of required stability, they are surprising in terms of the role of the knee musculature during stance. The knee joint, being primarily uni-axial, predominantly controls the height of the body's center of mass, whereas medio-lateral stability is controlled either at the hip with the abductor/adductor musculature or at the ankle with the invertor/evertor musculature. Thus, the process of postural modulation through sensory input must involve central mechanisms, invoking responses in muscles not underlying the area of stimulation.

In several previous studies, sub-threshold random stimulation, either in the form of vibration to the soles of the feet or in the form of electrical impulses to the knees or feet, has been demonstrated to reduce postural sway measures in both non-impaired young and old adults[31,41]. Of particular relevance is the study by Gravelle et al. (2002), who used a form of white noise electrical stimulation to the lateral aspects of the knee during one leg stance and demonstrated decreases in postural sway, as well as in the dispersion of the COP along the medio-lateral plane, similar in magnitude to those found in this study.

It is hypothesized that low-level noise enhances the detection and transmission of weak signals via a mechanism known as stochastic resonance[42]. This concept has been demonstrated in a variety of physical and biological systems, with human subjects demonstrating a lower somatosensory detection threshold as a 
result of noise application[43,44,45,46]. Thus, it is speculated that the application of noise enhances the use of undetected sub-threshold proprioceptive input that contributes to balance control.

There are two fundamental differences between the TENS used in the present study and electrical noise application, namely, the current stimulus parameters were constant rather than randomized, and intensity was set at the sensory detection level rather than at sub-threshold level. Therefore, it cannot be assumed that stochastic resonance is the underlying mechanism of the observed effect. It is more likely that the TENS enhances somatosensory input through its direct effect on the afferent nerve fibers. Experimental evidence indicates that the CNS is informed on the position and movements of the knee via ensemble coding mechanisms, rather than via modality specific pathways[47]. These coding mechanisms involve inputs from the skin, ligaments, capsular and muscle receptors, acting as a final common path that conveys information to the CNS[48]. Thus, although the effect of TENS is primarily exerted via cutaneous afferents, the potential influence on proprioceptive information to the CNS is highly plausible. As TENS inputs are mediated via central, mainly cortical, networks, which affect motor cortex excitability[20,49], higher-order effects may also contribute to the TENS-induced changes in postural sway. It must be, however, noticed that the small effect sizes (partial $\eta^{2}$ were below 0.10 ) are below what might be considered clinically useful. Yet, this preliminary study examined the effect of a very short stimulation period in young and healthy adults with intact postural stability. These factors most likely minimized the stimulation effects.

\section{CONCLUSIONS}

The results support our previous study and point to the potential of TENS applied at very low stimulation doses to affect postural sway, indicating that the location of the stimulus may have a directionally specific effect. Due to the limited nature of this study, however, wide-ranging interpretations should be avoided. Future studies should explore questions related to the clinical significance of these findings, particularly in populations with postural control impairments. The low cost and ease of application of TENS render its use extremely accessible. Additional research should also be conducted on various aspects of treatment protocol, such as stimulation parameters (e.g., application site and time, pulse width, frequency, and intensity), which may further enhance the effectiveness of treatment.

\section{REFERENCES}

1. Gurfinkel, V.S., Ivanenko Yu, P., Levik Yu, S., and Babakova, I.A. (1995) Kinesthetic reference for human orthograde posture. Neuroscience 68, 229-243.

2. Mergner, T., Maurer, C., and Peterka, R.J. (2003) A multisensory posture control model of human upright stance. Neural Control of Space Coding and Action Production 142, 189-199.

3. Kavounoudias, A., Roll, R., and Roll, J.P. (1998) The plantar sole is a 'dynamometric map' for human balance control. Neuroreport 9, 3247-3252.

4. Kavounoudias, A., Roll, R., and Roll, J.P. (2001) Foot sole and ankle muscle inputs contribute jointly to human erect posture regulation. $J$ Physiol May 1, 869-878.

5. Roll, R., Kavounoudias, A., and Roll, J.P. (2002) Cutaneous afferents from human plantar sole contribute to body posture awareness. Neuroreport 13, 1957-1961.

6. Stal, F., Fransson, P.A., Magnusson, M., and Karlberg, M. (2003) Effects of hypothermic anesthesia of the feet on vibration-induced body sway and adaptation. $J$ Vestib Res 13, 39-52.

7. Wu, G. and Chiang, J. (1997) The significance of somatosensory stimulations to the human foot in the control of postural reflexes. Exp Brain Res 114, 163-169.

8. Bloem, B.R., Allum, J.H.J., Carpenter, M.G., Verschuuren, J.j., and Honegger, F. (2002) Triggering of balance corrections and compensatory strategies in a patient with total leg proprioceptive loss. Experimental Brain Research 142, 91-107.

9. Koskimies, K., Sutinen, P., Aalto, H., Starck, J., Toppila, E., Hirvonen, T., Kaksonen, R., Ishizaki, H., Alaranta, H.., and Pyykko, I. (1997) Postural stability, neck proprioception and tension neck. Acta Oto-Laryngologica, 529, 95-97.

10. Vuillerme, N., Danion, F., Forestier, N., and Nougier, V. (2002) Postural sway under muscle vibration and muscle fatigue in humans. Neuroscience Letters 333, 131-135.

11. Horak, F.B. and Hlavacka, F. (2001) Somatosensory loss increases vestibulospinal sensitivity. Journal of 
Neurophysiology 86, 575-585.

12. Jeka, J.J., Easton, R.D., Bentzen, B.L., and Lackner, J.R. (1996) Haptic cues for orientation and postural control in sighted and blind individuals. Percept Psychophys 58, 409-423.

13. Dickstein, R., Shupert, C.L., and Horak, F.B. (2001) Fingertip touch improves postural stability in patients with peripheral neuropathy. Gait \& Posture 14, 238-247.

14. Bjordal, J.M., Johnson, M.I., and Ljunggreen, A.E. (2003) Transcutaneous electrical nerve stimulation (TENS) can reduce postoperative analgesic consumption. A meta-analysis with assessment of optimal treatment parameters for postoperative pain. European Journal of Pain (London, England) 7, 181-188.

15. Rushton, D.N. (2002) Electrical stimulation in the treatment of pain. Disabil Rehabil 24, 407-415.

16. Sluka, K.A. and Walsh, D. (2003) Transcutaneous electrical nerve stimulation: basic science mechanisms and clinical effectiveness. Journal of Pain 4, 109-121.

17. Garrison, D.W. and Foreman, R.D. (1994) Decreased activity of spontaneous and noxiously evoked dorsal horn cells during transcutaneous electrical nerve stimulation (TENS). Pain 58, 309-315.

18. Radhakrishnan, R. and Sluka, K.A. (2005) Deep tissue afferents, but not cutaneous afferents, mediate transcutaneous electrical nerve stimulation-induced anti hyperalgesia. Journal of Pain 6, 673-680.

19. Ridding, M.C., Brouwer, B., Miles, T.S., Pitcher, J.B., and Thompson, P.D. (2000) Changes in muscle responses to stimulation of the motor cortex induced by peripheral nerve stimulation in human subjects. Experimental Brain Research 131, 135-143.

20. Tinazzi, M., Zarattini, S., Valeriani, M., Romito, S., Farina, S., Moretto, G., Smania, N., Fiaschi, A., and Abbruzzese, G. (2005) Long-lasting modulation of human motor cortex following prolonged transcutaneous electrical nerve stimulation (TENS) of forearm muscles: evidence of reciprocal inhibition and facilitation. Experimental Brain Research 161, 457-464.

21. Potisk, K.P., Gregoric, M., and Vodovnik, L. (1995) Effects of transcutaneous electrical nerve stimulation (TENS) on spasticity in patients with hemiplegia. Scand J Rehabil Med 27, 169-174.

22. Tekeoglu, Y., Adak, B., and Goksoy, T. (1998) Effect of transcutaneous electrical nerve stimulation (TENS) on Barthel Activities of Daily Living (ADL) index score following stroke. Clin Rehabil 12, 277-280.

23. Armutlu, K., Meric, A., Kirdi, N., Yakut, E., and Karabudak, R. (2003) The effect of transcutaneous electrical nerve stimulation on spasticity in multiple sclerosis patients: a pilot study. Neurorehabil Neural Repair 17, 79-82.

24. Magnusson, M., Johansson, K., and Johansson, B.B. (1994) Sensory stimulation promotes normalization of postural control after stroke. Stroke 25, 1176-1180.

25. Tyson, S. (2003) Use of transcutaneous nerve stimulation to treat sensory loss after stroke. Physiother Res Int 8, 5357.

26. Guariglia, C., Coriale, G., Cosentino, T., and Pizzamiglio, L. (2000) TENS modulates spatial reorientantion in neglect patients. Neuroreport 11, 1945-1948.

27. Lafosse, C., Kerckhofs, E., Troch, M., and Vandenbussche, E. (2003) Upper limb exteroceptive somatosensory and proprioceptive sensory afferent modulation of hemispatial neglect. J Clin Exp Neuropsychol 25, 308-323.

28. Perennou, D.A., Leblond, C., Amblard, B., Micallef, J.P., Herisson, C., and Pelissier, J.Y. (2001) Transcutaneous electric nerve stimulation reduces neglect-related postural instability after stroke. Archives of Physical Medicine and Rehabilitation 82, 440-448.

29. van Dijk, K.R., Scherder, E.J., Scheltens, P., and Sergeant, J.A. (2002) Effects of transcutaneous electrical nerve stimulation (TENS) on non-pain related cognitive and behavioural functioning. Rev Neurosci 13, 257-270.

30. Dickstein, R., Laufer, Y., and Katz, M. (2006) TENS to the posterior aspect of the legs decreases postural sway during stance. Neuroscience Letters 393, 51-55.

31. Gravelle, D.C., Laughton, C.A., Dhruv, N.T., Katdare, K.D., Niemi, J.B., Lipsitz, L.A., and Collins, J.J. (2002) Noise-enhanced balance control in older adults. Neuroreport 13, 1853-1856.

32. Horak, F., Dimitrova, D., and Nutt, J. (2005) Direction-specific postural instability in subjects with Parkinson's disease. Exp Neurol. 193, 504-521.

33. Maki, B., Holliday, P., and Toppe, r.A. (1994) A prospective study of postural balance and risk of falling in an ambulatory and independent elderly population. J Gerontol. 49, 72-84.

34. Hafstrom, A., Fransson, P.A., Karlberg, M., Ledin, T., and Magnusson, M. (2002) Visual influence on postural control, with and without visual motion feedback. Acta Otolaryngol 122, 392-397.

35. Nagano, A., Yoshioka, S., Hay, D.C., Himeno, R., and Fukashiro, S. (2006) Influence of vision and static stretch of the calf muscles on postural sway during quiet standing. Hum Mov Sci 25, 422-434.

36. Raymakers, J.A., Samson, M.M., and Verhaar, H.J. (2005) The assessment of body sway and the choice of the stability parameter(s). Gait \& Posture 21, 48-58.

37. Hunter, M.C. and Hoffman, M.A. (2001) Postural control: visual and cognitive manipulations. Gait Posture , 41-48.

38. Adkin, A.L., Frank, J.S., Carpenter, M.G., and Peysar, G.W. (2000) Postural control is scaled to level of postural threat. Gait \& Posture 12., 87-93.

39. Laufer, Y., Barak, Y., and Chemel, I. (2006) Age-related differences in the effect of a perceived threat to stability on postural control. J Gerontol A Biol Sci Med Sci 61, 500-504.

40. Andersson, G., Hagman, J., Talianzadeh, R., Svedberg, A., and Larsen, H.C. (2002) Effect of cognitive load on postural control. Brain Res Bull 58, 135-139. 
41. Priplata, A., Niemi, J., Salen, M., Harry, J., Lipsitz, L.A., and Collins, J.J. (2002) Noise-enhanced human balance control. Phys Rev Lett 89, 238101.

42. Wiesenfeld, K., and Moss, F. (1995) Stochastic resonance and the benefits of noise: from ice ages to crayfish and SQUIDs. Nature 373, 33-36.

43. Collins, J.J., Imhoff, T.T., and Grigg, P. (1996) Noise-enhanced information transmission in rat SA1 cutaneous mechanoreceptors via aperiodic stochastic resonance. J Neurophysiol 76, 642-645.

44. Collins, J.J., Priplata, A.A., Gravelle, D.C., Niemi, J., Harry, J., and Lipsitz, L.A. (2003) Noise-enhanced human sensorimotor function. IEEE Eng Med Biol Mag 22, 76-83.

45. Dhruv, N.T., Niemi, J.B., Harry, J.D., Lipsitz, L.A., and Collins, J.J. (2002) Enhancing tactile sensation in older adults with electrical noise stimulation. Neuroreport 13, 597-600.

46. Liu, W., Lipsitz, L.A., Montero-Odasso, M., Bean, J., Kerrigan, D.C., and Collins, J.J. (2002) Noise-enhanced vibrotactile sensitivity in older adults, patients with stroke, and patients with diabetic neuropathy. Arch Phys Med Rehabil 83, 171-176.

47. Sjolander, P., Johansson, H., and Djupsjobacka, M. (2002) Spinal and supraspinal effects of activity in ligament afferents. J Electromyogr Kinesiol. 12, 167-176.

48. Hogervorst, T. and Brand, R.A. (1998) Mechanoreceptors in joint function. J Bone Joint Surg Am 80, $1365-1378$.

49. Deuchert, M., Ruben, J., Schwiemann, J., Meyer, R., Thees, S., Krause, T., Blankenburg, F., Villringer, K., Kurth, R., Curio, G., and Villringer, A. (2002) Event-related fMRI of the somatosensory system using electrical finger stimulation. Neuroreport 13, 365-369.

\section{This article should be cited as follows:}

Laufer, Y. and Dickstein, R. (2007) TENS to the lateral aspect of the knees during stance attenuates postural sway in young adults. TheScientificWorldJOURNAL: TSW Child Health \& Human Development 7, 1904-1911. DOI 10.1100/tsw.2007.279. 


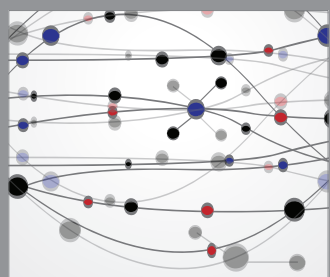

The Scientific World Journal
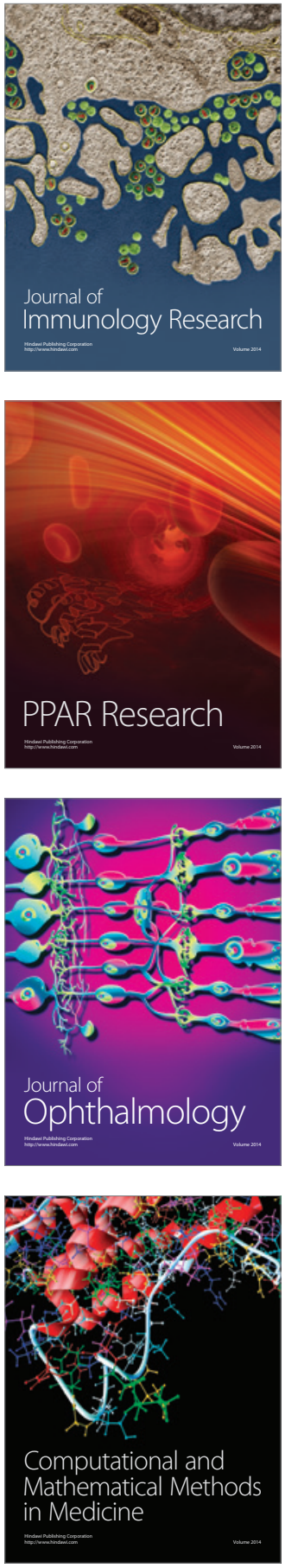

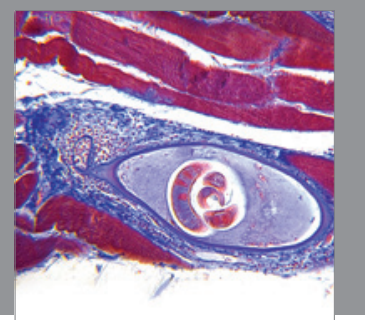

Gastroenterology

Research and Practice
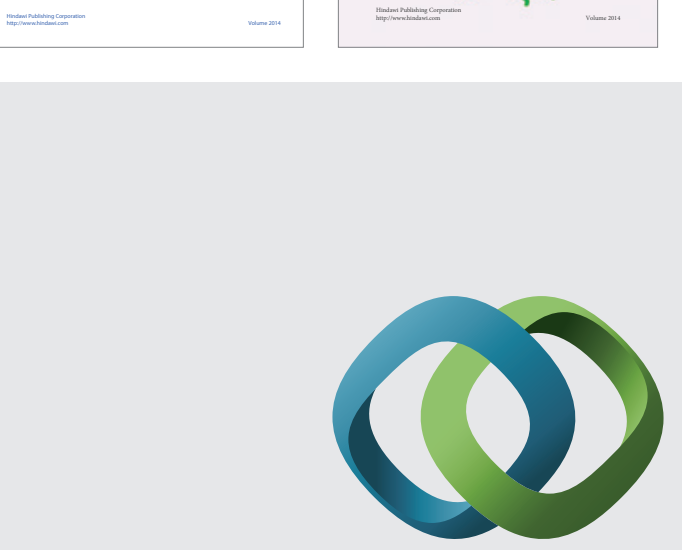

\section{Hindawi}

Submit your manuscripts at

http://www.hindawi.com
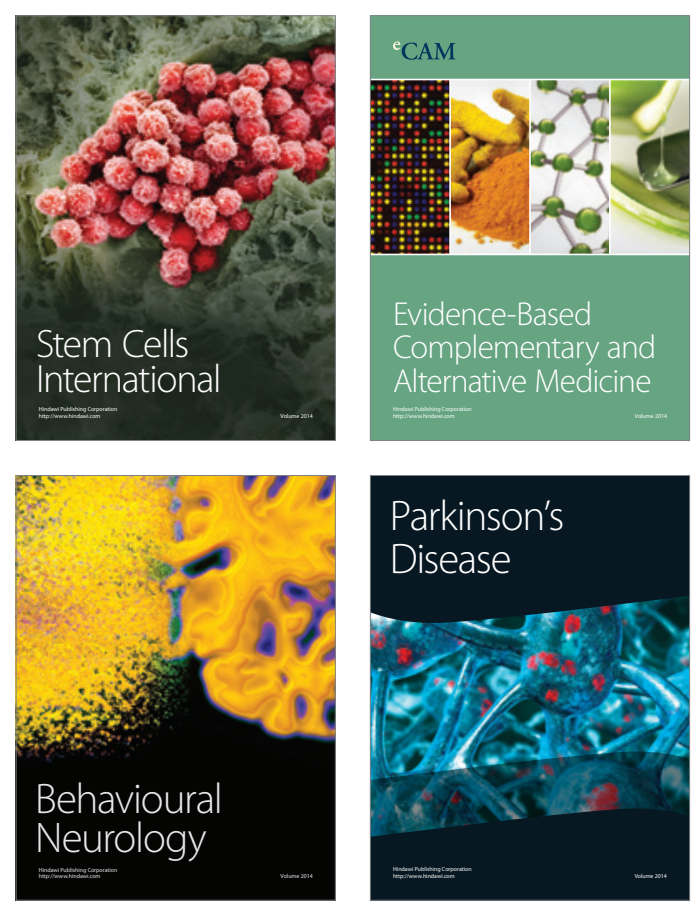

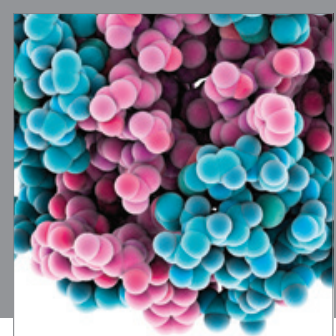

Journal of
Diabetes Research

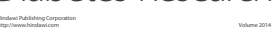

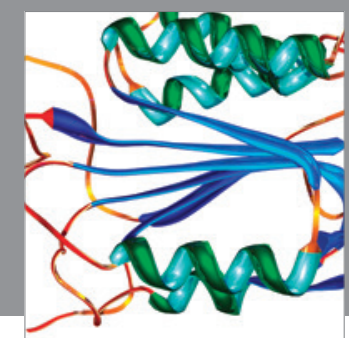

Disease Markers
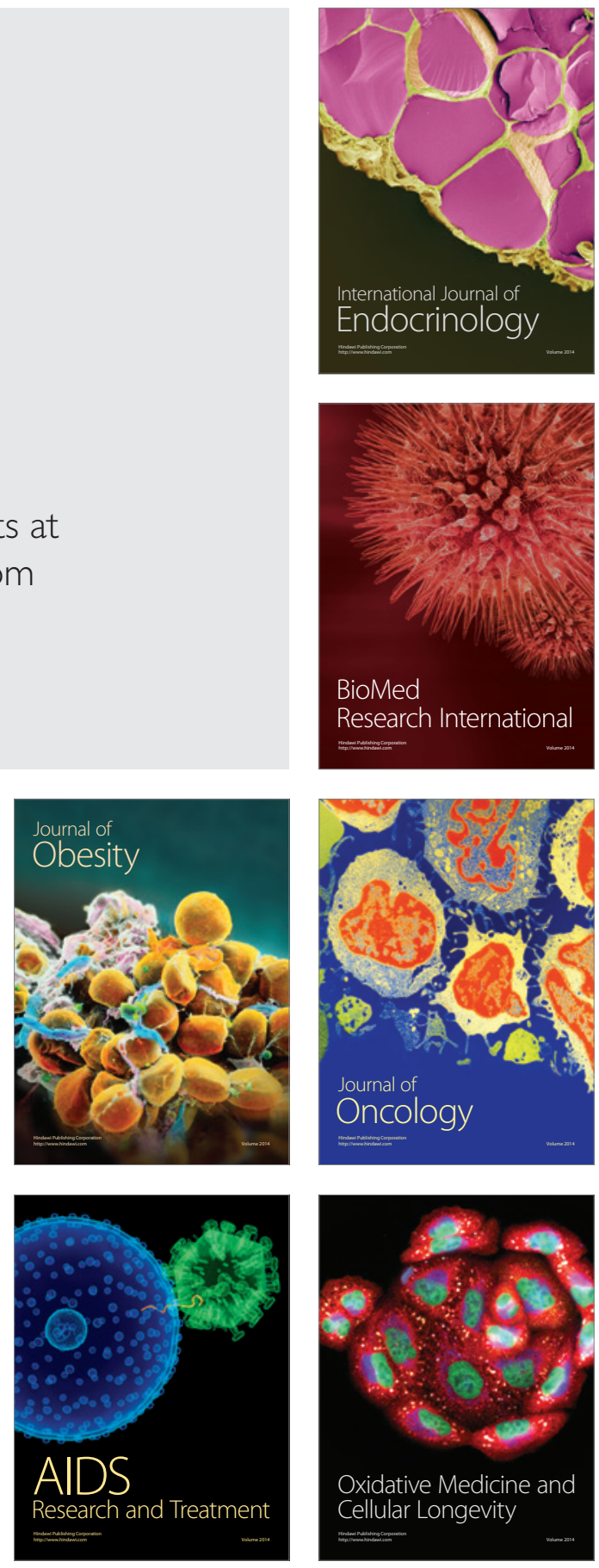